\title{
Cdk5 Regulates the Size of an Axon Initial Segment-Like Compartment in Mushroom Body Neurons of the Drosophila Central Brain
}

\author{
Svetlana Trunova, Brian Baek, and Edward Giniger \\ National Institute of Neurological Disorders and Stroke, National Institutes of Health, Bethesda, Maryland 20892
}

\begin{abstract}
The axon initial segment (AIS) is the specialized compartment of vertebrate axons where action potentials are initiated. Despite longtime attention to the unique functions of this compartment, the mechanisms that regulate AIS formation and maintenance are not known. Here, we identify a novel compartment in Drosophila mushroom body neurons that mirrors the molecular hallmarks of the vertebrate AIS as judged by accumulation of the anchoring protein Ankyrin1, presence of a specialized actin cytoskeleton, exclusion of both axonspecific and somatodendritic-specific cell surface proteins, and accumulation of a unique combination of voltage-gated ion channels. Using pharmacological treatments, we show that, similar to the vertebrate AIS, the integrity of this region of $\gamma$-neurons and its ability to tether membrane proteins depends on an intact actin cytoskeleton. We further show that $\mathrm{Cdk} 5 / \mathrm{p} 35$ kinase regulates the formation and maintenance of the putative AIS by controlling the position of its distal boundary. Thus, boosting Cdk 5 activity in $\gamma$-neurons extends the AIS by as much as $100 \%$, while eliminating Cdk 5 activity causes the domain to shrink proximally or disappear altogether. These data demonstrate that Cdk5/p35 kinase is a key regulator of the development and maintenance of the AIS in Drosophila.
\end{abstract}

\section{Introduction}

The axon initial segment (AIS) is the specialized domain of vertebrate neurons that lies between the somatodendritic and axonal compartments, restricting diffusion of membrane proteins and lipids and regulating cytoplasmic transport (Nakada et al., 2003; Hedstrom and Rasband, 2006; Sobotzik et al., 2009). Because of its unique population of voltage-gated ion channels (Ogawa and Rasband, 2008; Lasiecka et al., 2009), it is also the site of action potential initiation (Kole et al., 2008), and consequently it is a key site for regulating plasticity (Grubb and Burrone, 2010; Kuba et al., 2010). Recently, a link between AIS function and neuronal injury was established, with various neuronal insults leading to calpain-dependent disruption of AIS structure and function (Schafer et al., 2009; von Reyn et al., 2009). Despite the extensive literature on AIS properties, the mechanisms that control its development and maintenance remain largely unknown. The organization of the AIS is only known to depend on AnkyrinG, which

Received Jan. 7, 2011; revised May 13, 2011; accepted June 2, 2011.

Author contributions: S.T. and E.G. designed research; S.T. and B.B. performed research; S.T. and E.G. analyzed data; S.T. and E.G. wrote the paper.

This work was supported by the Basic Neuroscience Program of the NINDS Intramural Research Program, NIH Grant Z01 NS003106. We thank Morgan Sheng and Bruce Tempel for helpful discussion; Stephen Wincovitch for help with confocal microscopy; and Ben White, Mark Stopfer, Julie Simpson, Chi-hon Lee, and the Giniger Laboratory for comments on this manuscript. We also thank Ben White, Ron Dubreuil, Liqun Luo, Yasushi Hiromi, and Andreas Prokop for providing reagents and especially thank Julie Simpson for sharing unpublished Elk and Shal transgenic strains.

The authors declare no competing financial interests.

Correspondence should be addressed to Edward Giniger, National Institute of Neurological Disorders and Stroke, National Institutes of Health, 35 Convent Drive, Room 1C1002, Bethesda, MD 20892. E-mail: ginigere@ ninds.nih.gov.

DOI:10.1523/JNEUROSCI.0117-11.2011

Copyright $\odot 2011$ the authors $\quad 0270-6474 / 11 / 3110451-12 \$ 15.00 / 0$ localizes AIS components by binding them to the underlying cytoskeleton (Hedstrom and Rasband, 2006). Nevertheless, the existence of microdomains in the AIS and AnkG-independent accumulation of some cell surface proteins imply AnkGindependent aspects of AIS assembly (Winckler et al., 1999; Van Wart et al., 2007). Establishment of a simple, genetically tractable in vivo model would help to uncover the molecular mechanisms that shape the AIS.

One component that is essential for neuronal structure and physiology is the cyclin-dependent kinase, Cdk5. Cdk5 is composed of a broadly expressed catalytic subunit, CDK5, and, in vertebrates, one of two paralogous regulatory subunits, $p 35$ and p39 (Ko et al., 2001). Within the nervous system, Cdk5 has been implicated in fidelity of axon patterning and lamination of the mammalian cortex during development, and synaptic function, neurotransmission, learning, and memory in the adult (Ip and Tsai, 2008). Moreover, Cdk5 function is associated with many human neurodegenerative diseases (Ip and Tsai, 2008). Nevertheless, the essential roles of Cdk5 in normal physiology, and in the transition from normal function to neurodegeneration, remain unclear. We therefore set out to analyze Cdk5 functions in mature, differentiated neurons in vivo, using mushroom body (MB) $\gamma$-neurons of the Drosophila central brain as a model. In Drosophila, unlike mammals, mutations inactivating Cdk5 activity are viable and do not cause defects in neuronal positioning. Moreover, there is only a single p35 in the fly, and no p39-like paralog, simplifying analysis of its function (Connell-Crowley et al., 2007).

We here show that Drosophila $\gamma$-neurons have a domain in the proximal axon that shares many of the key hallmarks of the vertebrate AIS, including accumulation of Ankyrin1, segregation of 
the axonal and somatodendritic membranes, and accumulation of a unique constellation of voltage-gated ion channels. Pharmacological treatments reveal a role for the actin cytoskeleton in integrity of this region and its ability to tether membrane proteins. We further show that $\mathrm{Cdk} 5 / \mathrm{p} 35$ protein kinase is a key regulator of AIS size, as decreased activity of Cdk5 leads to virtual disappearance of the AIS-like compartment, while increased activity nearly doubles its length.

\section{Materials and Methods}

Fly stocks and genetics

Bloomington Center provided the following stocks: UAS-actin-GFP, UAS-Syt-GFP, UAS- $a$-tub-GFP; hsFLP122, UAS-mCD8-GFP;Gal80, FRT40A,201YGal4, UAS-mCD8/CyO, FRT40A, and 201YGal4 were kindly provided by Dr. L. Luo (Stanford University, Palo Alto, CA); NP21-Gal4 by Dr. K. Ito (University of Tokyo, Tokyo, Japan); UAS-RdlHA/FM7 and UAS-Syt-HA by Dr. A. Prokop (University of Manchester, Manchester, UK); UAS-Robo2-GFP, UAS-Robo3-GFP, and UAS-DrlGFP by Dr. Y. Hiromi (NIG, Shizuoka, Japan); UAS-DRK-C2-GFP, UAS-Shaw, UAS-EKO-GFP by Dr. B. White (NIH, Bethesda, MD); UASAnk1-GFP and UAS- $\beta$-spectrin-myc by Dr. R. Dubreuil (University of Illinois, Chicago, Chicago, IL); and UAS-elk-GFP and UAS-Shal-HA by Dr. J. Simpson (Janelia Farm, Ashburn, VA).

Null alleles $p 35^{20 c}$ and DfC2 and insertions UAS-Cdk5DN-FLAG, UASCdk5-FLAG, and UAS-p35-myc were generated previously (ConnellCrowley et al., 2000). In all our experiments, $p 35^{20 c}$ homozygote and $p 35^{20 c}$ / $D f C 2$ transheterozygote had identical phenotypes.

Mosaic analysis with a repressible cell marker genotypes. Mosaic analysis with a repressible cell marker (MARCM) genotypes were as follows: $h s$ Flp122, UAS-mCD8-GFP; UAS-mCD8, GAL4-201Y, FRT40A/FRT40A tubP-GAL80 crossed to w; FRT40A males (Lee et al., 1999); hs-Flp122, UAS-mCD8-GFP/Y; UAS-mCD8, GAL4-201Y, p35 $5^{20 c}$, FRT40A/FRT40A tubP-GAL80 crossed to $w$; $p 35^{20 c}$, FRT40A males.

Flp-out genotypes. Flp-out genotypes were as follows: hs-FLP; $U A S>C D 2, y+>m C D 8$-GFP crossed to $w$; OK107Gal4 males (Wong et al., 2002); $h s-F L P ; p 35^{20 c}$; UAS $>C D 2, y+>m C D 8$-GFP crossed to $w$; p $35^{20 c}$; OK107Gal4 males.

All flies were raised on standard media at $25^{\circ} \mathrm{C}$ and kept at low to medium density. All experiments were performed with males, except UAS-DORK-C2-GFP flies, which were kept at $18^{\circ} \mathrm{C}$ and included analysis of both females and males due to very low viability. MARCM and FLPout clones were generated by heat shocking first-instar larvae at $37^{\circ} \mathrm{C}$ for $5 \mathrm{~min}$.

\section{Immunohistochemistry}

Immunostaining was performed as described previously (Lee et al., 1999) with some modifications for larval and adult brains. Middle third-stage larval brains were dissected in PBS, pH 7.4 (Invitrogen), and immediately fixed in 3.7\% PFA in PBS for 25 min and postfixed in 3.7\% PFA in PBS with $0.5 \%$ Triton X-100 for $25 \mathrm{~min}$. After several washes in PBS buffer with $0.5 \%$ Triton X-100 (PBST) for $2 \mathrm{~h}$ brains were blocked in block buffer (PBST, $0.5 \%$ BSA, 5\% goat serum) for $1 \mathrm{~h}$ and incubated with primary antibodies in block buffer overnight at $4^{\circ} \mathrm{C}$. Primary antibodies were as follows: mouse anti-Fasciclin 2 (Fas2) and anti- $\alpha$-spectrin, rat anti-N-Cad from Developmental Studies Hybridoma Bank, chicken anti-GFP (Aves), rabbit anti-myc and anti-FLAG (Sigma-Aldrich), and rat anti-HA (Roche). Secondary antibodies were as follows: goat antimouse, anti-rabbit, and anti-rat conjugated with A488, A568, A633 fluorochromes (Invitrogen) were used at 1:500 dilution in block buffer, and donkey anti-rat-FITC (Jackson ImmunoResearch) at 1:300 dilution.

Then brains were washed in PBST for $2 \mathrm{~h}$ and incubated with secondary antibodies for $4 \mathrm{~h}$ and afterward washed for $2 \mathrm{~h}$ more in PBST. Samples were equilibrated for $2 \mathrm{~h}$ and mounted in Bio-Rad antifade medium. To prevent deformation of samples, glass chips (no. 1) were placed between coverslips and slides. Adult brains were processed similarly with slight modifications. All treatment times were increased: fixation time was $70 \mathrm{~min}$ total, primary antibody incubation time $48 \mathrm{~h}$ at $4^{\circ} \mathrm{C}$, and secondary antibody was added for $12-16 \mathrm{~h}$ at $4^{\circ} \mathrm{C}$.

\section{Live brain culturing}

Live extraction protocol was adopted from that of Winckler et al. (1999) and adjusted to preserve morphology of Drosophila brains. Brains were individually dissected from middle third-stage wild-type larvae in PBS and immediately transferred to Schneider's Drosophila Medium (Invitrogen) supplemented with $10 \%$ inactivated FCS, $0.4 \%$ penicillin/streptomycin, and $20 \mu \mathrm{g} / \mathrm{ml}$ cycloheximide (Sigma-Aldrich) $(50 \mathrm{mg} / \mathrm{ml}$ stock in DMSO); brains were cultured at $25^{\circ} \mathrm{C}$ for $16 \mathrm{~h}$, media was replaced every $8-10 \mathrm{~h}$. Latrunculin B (LatB) $(0.4 \mu \mathrm{g} / \mathrm{ml})$ (Calbiochem) $(200$ $\mu \mathrm{g} / \mathrm{ml}$ stock in ethanol) and cytochalasin B (CytB) $(20 \mu \mathrm{M})$ (Calbiochem) (10 mM stock in ethanol) or 1\% (v/v) DMSO (Sigma-Aldrich) were added simultaneously with medium. To prevent actin polymerization, we applied combination of $0.4 \mu \mathrm{g} / \mathrm{ml}$ latrunculin $\mathrm{B}$ and $20 \mu \mathrm{M}$ cytochalasin B (LatB/CytB). For live detergent extraction assay, one-half of the samples after culturing for $16 \mathrm{~h}$ were extracted in $0.5 \%$ CHAPS (Sigma-Aldrich) (50\% stock in $\mathrm{H}_{2} \mathrm{O}$ ) with matching culturing media at $25^{\circ} \mathrm{C}$. After treatments, brains were immediately fixed in $4 \% \mathrm{PFA} / 3 \%$ sucrose in PBS for $25 \mathrm{~min}$ and postfixed for $25 \mathrm{~min}$ in the same fixative with $0.2 \%(\mathrm{w} / \mathrm{v})$ Tween; staining protocol is shown above where $0.5 \%$ Triton was replaced by $0.2 \%$ Tween in all applications. Suppression of biosynthesis was confirmed by absence of Fas2-positive vesicles in soma, reduced level of N-Cad and neuroglian signal in the soma. For quantification, the two mushroom bodies from each brain were scored separately. DMSO treatment alone produced results very similar to controls under normal culturing conditions and after CHAPS extraction (data not shown).

All images were taken using identical microscope settings. Since destabilization of F-actin with LatB/CytB and CHAPS treatment significantly damaged the brain tissue, MBs were first analyzed under higher level of light intensity and identified using MB reporter construct, Fas2, and NCad patterns.

\section{Image analysis}

Samples were imaged on a Zeiss LSM 510 confocal microscope; images were processed using AimImageBrowser (Zeiss) for presentation purposes and Surpass View application on IMARIS software for AIS size quantification in $Z$-stacks. Using IMARIS Surpass application, we traced and measured neuronal projections in three dimensions in whole-mount brain samples. All measurements were done for late third larval stage males when brains reach their maximum size and 5-7 d adult males.

Because $\gamma$-neuron cell bodies are at significant and variable distance from the start of the axonal compartment, we defined a uniform zero reference point for measurements at the end of the dendrite emergence zone by focusing through each image stack, and marking the most distal point at which the bundle of dendritic projections that form the calyx intersects the fascicle of Kenyon cell axons from the most lateral MB neuroblast. Regarding the boundaries of compartment-specific markers, accumulation of compartment-specific markers was graded, not abrupt (Winckler et al., 1999; Nakada et al., 2003). Therefore, we manually defined the position of the compartment border as the middle of the staining gradient in the most lateral bundle of $\gamma$-neuron projections. Surpass View application on IMARIS software can trace and measure true three-dimensional length of neuronal projections in whole-mount brain samples.

\section{Results}

\section{Morphology of the Drosophila mushroom body neurons}

The MB is the center of olfactory learning and memory in the fly and is composed of several types of neurons that are produced in a stereotyped manner (Lee et al., 1999; Kurusu et al., 2002). The MB neurons have a unipolar morphology, with the dendritic arbor sprouting from a proximal part of the axon. The neurites from the progeny of each of four precursor neuroblasts fasciculate to form a bundle and project their dendrites into the calyx, while the axons proper project into a peduncle that splits into various lobes (Fig. 1I). The order of projection inside the peduncle and their projection into the five adult lobes depends on neuronal type; $\gamma$-neurons are generated first and by the late third 

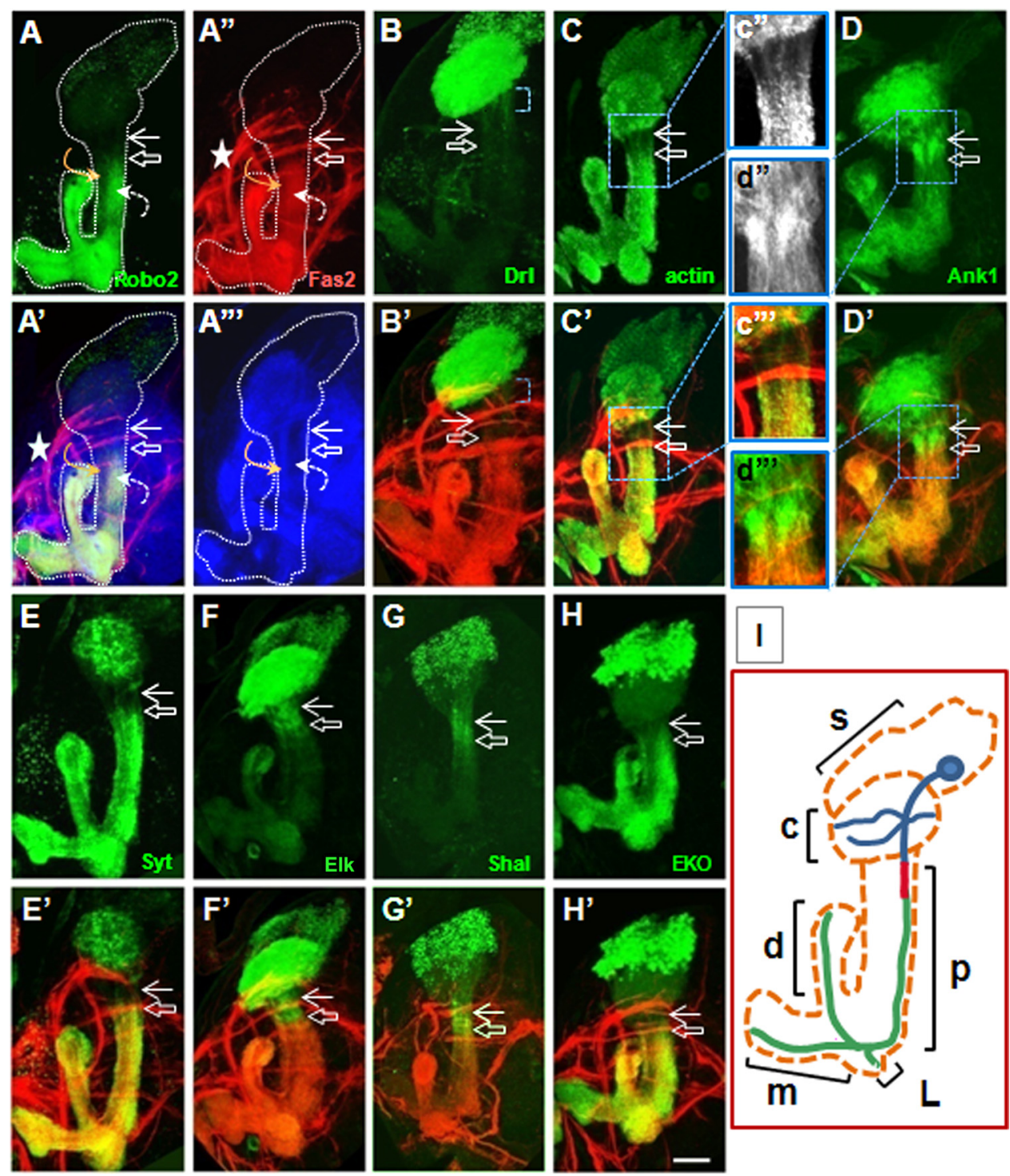

Figure 1. Drosophila $\gamma$-neurons develop a unique intraaxonal compartment analogous to the vertebrate AIS. Flies were generated by crossing 201YGal4 females with males containing UAS transgene in all following figures. Composite confocal images of late third-instar larval brains show the entire MB. All MBs are oriented identically where cell bodies are shown up, dendrites project into the calyx area below, peduncle is in the middle, dorsal lobe projects from right to left, and middle lobes project to the left. Fas 2 staining is shown in red, relevant UAS construct in green, and NCad in blue. The top rows $(\boldsymbol{A}-\boldsymbol{H})$ show pattern of individual markers, and the bottom rows are merged images $\left(\boldsymbol{A}^{\prime}-\boldsymbol{H}^{\prime}\right)$. Robo2-GFP $(\boldsymbol{A})$ and Fas2 $\left(\boldsymbol{A}^{\prime \prime}\right)$ label the axonal and Drl-GFP $(\boldsymbol{B})$ labels the somatodendritic compartments. The hollow white arrow shows position of axon proximal boundary, and the solid white arrow points to distal boundary of somatodendritic part. Single optic slice $(1.5 \mu \mathrm{m})$ shows NCad pattern through the middle part of peduncle $\left(\boldsymbol{A}^{\prime \prime \prime}\right)$. The curved orange arrow points to the axons of $\alpha-, \beta$-neurons projecting in the inner layer of peduncle and the dashed curved white arrow points to outer layer of peduncle containing axons of $\gamma$-neurons $\left(\boldsymbol{A}-\boldsymbol{A}^{\prime \prime \prime}\right)$. NCad is evenly distributed throughout $\gamma$-neurons $\left(\boldsymbol{A}^{\prime}, \boldsymbol{A}^{\prime \prime \prime}\right)$ and has lower expression levels in $\gamma$-neurons compared with $\alpha, \beta$-neurons. The white dotted line outlines the entire MB as identified with Fas 2 and NCad markers. Note the position of inner antennocerebral tract in direct contact with calyx $\left(A^{\prime \prime}\right.$, asterisk). Drl-GFP is present in dendritic arbor and proximal neurite beyond calyx (B, blue bracket). Actin-GFP ( $\boldsymbol{C}$ ) and Syt-HA $(\boldsymbol{E})$ are excluded, and Ank1-GFP (D), Elk-GFP (F), and Shal-myc (G) are accumulated in interstitial domain between axonal and dendritic compartments. Tub-GFP did not show altered distribution in this region. The insets $\left(\boldsymbol{C}^{\prime \prime}, \boldsymbol{d}^{\prime \prime}\right)$ show a higher magnification of AlS area. $\boldsymbol{H}$, EKO-GFP is concentrated in the axonal and interstitial compartments, and in a dorsal part of the calyx. Scale bar, $30 \mu \mathrm{m}$. I, Schematic showing structure and position of single $\gamma$-neurons inside mushroom body; $s$ (soma) and c (calyx) represent somatodendritic compartment shown in blue for $\gamma$-neuron; $p$ (peduncle) includes Als compartment shown in red and proximal part of axon in green; $d$ (dorsal), $m$ (medial), and L (lateral) axon lobes.

larval stage $\gamma$-neurons are fully differentiated and project their axons and dendrites to the appropriate target areas (Lee et al., 1999). Morphology of these neurons was assayed using the Gal4/ UAS system of targeted gene expression, visualizing MB $\gamma$-neurons using the 201YGal4 or OK107-Gal4 drivers.

\section{Drosophila CNS neurons develop a unique intraaxonal compartment}

In the process of examining subcellular organization of wild-type $\gamma$-neurons at late larval stage, we noted a large and varied selec- tion of markers whose localization suggested the existence of a short, unique domain in the proximal axon, lying between the somatodendritic and axonal compartments but distinct from both. All the strictly axonal membrane protein markers that we examined [Fas2 (Silies and Klämbt, 2010), ROBO2-GFP, ROBO3-GFP (Kurusu et al., 2002; Katsuki et al., 2009; Silies and Klämbt, 2010)] labeled the distal axon but not the part most proximal to the cell body. All of these markers shared a common boundary in the proximal axon (Fig. $\left.1 A, A^{\prime \prime}\right)$. To quantify the location of this boundary, a zero point for length measurements 
was defined at the end of the domain of dendrite emergence, and the distance along the axon was measured to the border of accumulation of the relevant marker (for details, see Materials and Methods). By this method, the proximal border of accumulation of axonal markers was mapped $41 \pm 7 \mu \mathrm{m}$ (mean $\pm \mathrm{SD}$ ) distal to the site of dendrite emergence (Fig. 2). The point of dendrite emergence was identified by staining for the general markers $\mathrm{N}$-Cadherin or F-actin. Fas2 was used as an endogenous internal marker for the border of the axonal domain in all experiments described below.

The distal boundary of the somatodendritic compartment in $\gamma$-neurons was mapped by expression of the tagged, dendrite-specific markers RDL-HA, encoding the $\mathrm{GABA}_{\mathrm{A}}$ receptor, and the guidance receptor DRL-GFP, and by staining for the endogenous protein Futsch (the Drosophila MAP1B) and the endogenous epitope recognized by mAb BP102 (Sánchez-Soriano et al., 2005; Katsuki et al., 2009). All these markers were enriched in the calyx (i.e., dendrites). DRL-GFP (Fig. $1 B$ ), BP102, and Futsch were also present in the soma, and in the proximal neurite between the soma and the point of dendrite emergence (Table 1). Following the nomenclature of Gouwens and Wilson (2009), we refer to this region as the "primary neurite." In the case of DRL-GFP [and also dORKC2-GFP (see Fig. $4 \mathrm{~V}$ ) (see below)], somatodendritic GFP signal continued $26 \pm 7 \mu \mathrm{m}$ beyond the dendrite emergence zone (defining the border of the domain as the middle of a short, graded transition zone) (Fig. 2). For the purpose of this study, we defined the distal border of the somatodendritic compartment based on the boundary of accumulation of DRL-GFP in the primary neurite, beyond the dendrite emergence zone.

Crucially, the somatodendritic signal did not extend all the way to the proximal border of the axonal domain. Rather, there was an interstitial region of $\sim 16 \pm 7 \mu \mathrm{m}$ between the apparent borders of the somatodendritic and axonal compartments that did not possess the markers of either. This is apparent, for example, as an unlabeled zone in preparations double-stained for a GFP-tagged somatodendritic marker and for the endogenous Fas2 (Fig. $1 B^{\prime}$, region between arrows).

Cytoplasmic markers belonging to a different class of molecules, synaptic proteins, revealed a distribution pattern distinct from those of the axonal and somatodendritic markers but respecting the same borders when expressed in $\gamma$-neurons. Synaptotagmin (Syt) is a protein of the presynaptic terminal (SánchezSoriano et al., 2005). In larval $\gamma$-neurons, Syt-GFP was found both in the somatodendritic and axonal compartments but was selectively excluded from the unique region between them, creating a "clear zone" that was $27 \pm 3 \mu \mathrm{m}$ long (Fig. $1 E$ ). Notably, the distal border of the clear zone essentially coincided with the proximal border of Fas2 localization in double-labeled preparations (Fig. $1 E^{\prime}$ ). We confirmed this unusual distribution by expressing Syt-GFP with different MB-specific Gal4 drivers (NP21-Gal4 and OK-107) and also by expressing Syt tagged with HA under 201Y-Gal4. The same pattern was also observed for a second presynaptic marker, Bruchpilot-GFP (data not shown). It was necessary to examine expressed, tagged marker proteins in this experiment since localization of the endogenous proteins was obscured by their ubiquitous expression in surrounding cells.
To understand whether this unique interstitial region had some structural properties that could explain the observed distribution of membrane and synaptic vesicle proteins, we analyzed the morphology of the neuronal cytoskeleton. Expression of actin-GFP revealed a distribution that correlated with that of both synaptic vesicle proteins (Fig. 1C). The level of actin-GFP was significantly reduced in a band of $24 \pm 8 \mu \mathrm{m}$ that coincided with the Syt clear zone. In this region, actin-GFP gave the appearance of a bundle of parallel fibrils (Fig. $1 c^{\prime \prime}$ ). Thus, this domain has a specialized F-actin cytoskeleton, lacks both axonal and somatodendritic markers, and excludes synaptic vesicle proteins.

Recent work on unipolar projection neurons of the Drosophila antennal lobe demonstrated that the action potential is generated in a proximal part of the axon, distal to the point of dendrite emergence (Gouwens and Wilson, 2009). The apparent spatial coincidence of this physiologically identified spike initiation zone with the specialized domain we identified in MB neurons led us to speculate whether this unique interstitial axonal region might represent a compartment analogous or homologous to the vertebrate axon initial segment.

\section{$\boldsymbol{\gamma}$-neurons have an AIS-like compartment}

If the interstitial axonal region in Drosophila $\gamma$-neurons is homologous to the mammalian AIS we would predict (1) local enrichment of ankyrin, (2) presence of a specialized F-actin cytoskeleton, and (3) accumulation of a unique combination of voltage-gated ion channels.

(1) A landmark feature of the mammalian AIS is accumulation of an anchoring complex containing AnkG/ $\beta \mathrm{IV}$-spectrin. Drosophila does not have an AIS-specific AnkG/Ank3 gene, only the ubiquitous Ank1 and the neuron-specific Ank2 (Bouley et al., 2000). Anti-ankyrin antibodies were not available but expressed Ank1-GFP was clearly enriched in the putative AIS-like compartment (Fig. $\left.1 D, d^{\prime \prime}\right)$. Note that we expect only enrichment in this region, and not exclusive localization, since some ankyrin must be present throughout the axon (Bouley et al., 2000; Pielage et al., 2008). We estimated the length of the Ank1-positive region as $18 \pm 3 \mu \mathrm{m}$ (Fig. 2) coinciding well with the position and size of the specialized F-actin zone and abutting the proximal edge of the Fas2-positive domain in double-labeled samples (Fig. $1 D^{\prime}, d^{\prime \prime \prime}$ ). Both endogenous $\alpha$-spectrin and expressed $\beta$-spectrin-myc were present in the putative AIS, although neither was enriched (data not shown).

(2) Accumulation of AnkG in the mammalian AIS is supported by a tethered actin cytoskeleton with altered sensitivity 
Table 1. Endogenous and ectopic markers are targeted to distinct compartments in $\gamma$-neurons

\begin{tabular}{|c|c|c|c|c|c|c|}
\hline Marker & Soma & Dendrites & AIS & Peduncle & Axon lobes & Source \\
\hline DLG & + & +++ & + & +++ & +++ & DSHB \\
\hline BP104 & + & +++ & + & +++ & ++++ & DSHB \\
\hline BP102 & + & +++ & + & + & ++ & DSHB \\
\hline BP106 & + & +++ & + & +++ & ++ & DSHB \\
\hline $22 C 10$ & + & + & - & - & - & DSHB \\
\hline Fas2 & - & - & - & + & + & DSHB \\
\hline a-Spectrin & ++ & +++ & +++ & +++ & +++ & DSHB \\
\hline Fascin & + & + & + & + & + & DSHB \\
\hline Endophilin & +++ & +++ & + & +++ & +++ & Dr. Bellen \\
\hline KCNQ2 & + & + & + & + & + & Dr. Levitan \\
\hline Actin-GFP & +++ & +++ & + & +++ & +++ & BDSC \\
\hline GMA-GFP & +++ & +++ & + & +++ & +++ & Dr. Keihart \\
\hline Tubulin-GFP & +++ & +++ & +++ & +++ & +++ & BDSC \\
\hline Tau-myc & +++ & ++ & ++ & ++ & & BDSC \\
\hline mCD8-GFP & +++ & +++ & +++ & +++ & +++ & Dr. Luo \\
\hline Ankyrin1-GFP & + & +++ & +++ & + & + & Dr. Dubreuil \\
\hline b95-spectrin-myc & ++ & +++ & +++ & +++ & +++ & Dr. Dubreuil \\
\hline Nod-LacZ & +++ & ++ & - & - & - & Dr. Jan \\
\hline Rdl-HA & +++ & ++ & - & - & - & Dr. Prokop \\
\hline Homer-myc & +++ & ++ & ++ & ++ & ++ & Dr. Prokop \\
\hline APC2-GFP & +++ & +++ & - & ++ & - & Dr. Pfeifer \\
\hline Baz-GFP & +++ & +++ & - & + & - & Dr. Prokop \\
\hline Brp-GFP & +++ & + & - & + & + & Dr. Sigrist \\
\hline Syt-GFP & + & +++ & + & +++ & +++ & BDSC \\
\hline Syt-HA & + & +++ & + & +++ & +++ & Dr. Prokop \\
\hline Drl-GFP & + & +++ & - & - & - & Dr. Hiromi \\
\hline Robo2-GFP & + & - & - & +++ & +++ & Dr. Hiromi \\
\hline Robo3-GFP & ++ & - & - & +++ & +++ & Dr. Hiromi \\
\hline Robo-GFP & ++ & +++ & ++ & ++ & ++ & Dr. Hiromi \\
\hline Shaw-DN-FLAG & +++ & + & ++ & + & + & Dr. White \\
\hline Shaw-FLAG & +++ & +++ & +++ & +++ & +++ & Dr. White \\
\hline ShakerDN-SDT301-GFP & +++ & + & + & +++ & +++ & Dr. White \\
\hline dORK-C2-GFP & ++ & +++ & + & + & + & Dr. White \\
\hline dORK-NC1-GFP & +++ & + & + & - & - & Dr. White \\
\hline Elk-GFP & ++ & +++ & ++ & - & - & Dr. Simpson \\
\hline Shal-myc & +++ & + & ++ & - & - & Dr. Simpson \\
\hline Shal-HA & +++ & + & ++ & - & - & Dr. Simpson \\
\hline Sei-HA & +++ & + & + & - & - & Dr. Simpson \\
\hline
\end{tabular}

DSHB, Developmental Studies Hybridoma Bank; BDSC, Bloomington Drosophila Stock Center. Relative signal level in different neuronal compartments is shown as follows: " + + " for strong, " + + for moderate, "+ "for low signal, and "-" for no signal detected.

Suppliers: H. Bellen, Baylor College of Medicine, Houston, TX; I. Levitan, Thomas Jefferson University, Philadelphia, PA; D. Kiehart, Duke University, Durham, NC; Y. N. Jan, University of California, San Francisco, San Francisco, CA; B. Pfeiffer, Janelia Farm, Ashland, VA; S. Sigrist, FU Berlin, Berlin, Germany.

to actin depolymerizing agents (Winckler et al., 1999). As shown above, the Drosophila AIS-like compartment has an actin structure that is morphologically distinguishable from those of the adjacent somatodendritic and axonal compartments. To investigate the state of the actin cytoskeleton in the AIS, we treated larval brains in culture with LatB, which blocks actin polymerization. Treatment with LatB substantially reduced actin-GFP accumulation in dendrites and axons but did not have any visible effect in the AIS area, causing the distribution of actin-GFP to become uniform throughout all neurites. Thus, reducing polymerization of actin revealed a stable state of the actin cytoskeleton in the AIS-like compartment (Fig. 3A,B).

(3) The key physiological feature of the mammalian AIS is the accumulation of a unique constellation of voltage-gated sodium and potassium channels responsible for generation of action potentials (Ogawa and Rasband, 2008). There are no available antibody or transgenic reagents for localizing sodium channels in Drosophila neurons in situ. However, we expressed eight tagged versions of six potassium channels and found that seven of them showed boundaries in the patterns of accumulation that coincided with the borders of the putative AIS. Elk-GFP and ShawDN-FLAG (Hodge et al., 2005) accumulated in the AIS and were present in the somatodendritic compartments, as did Shal-myc and Shal-HA (Fig. 1F, G). dORK-C2-GFP (Nitabach et al., 2002) accumulated in dendrites and the primary neurite but was selectively excluded from the AIS (Fig. $4 \mathrm{~V}$ ), whereas the Shaker-DNGFP and its derivative EKO-GFP (White et al., 2001; Mosca et al., 2005) colocalized with axonal markers but were excluded from the AIS (Fig. $1 H$, Table 1), in good agreement with the previously published axonal distribution of Sh in motoneurons (Ryglewski and Duch, 2009). In each case, the border of accumulation of the channel essentially coincided with one of the two borders of the putative AIS.

Thus, all three landmark properties of the mammalian AIS are preserved in the interstitial axonal domain of Drosophila $\gamma$-neurons, supporting the hypothesis that this represents an AISlike axonal compartment.

\section{A dynamic F-actin cytoskeleton controls polarized retention of Fas2}

In the mammalian AIS, proper dynamics of the actin cytoskeleton are essential for maintaining the appropriate localization of tethered membrane proteins, such as NF (neurofilament), 

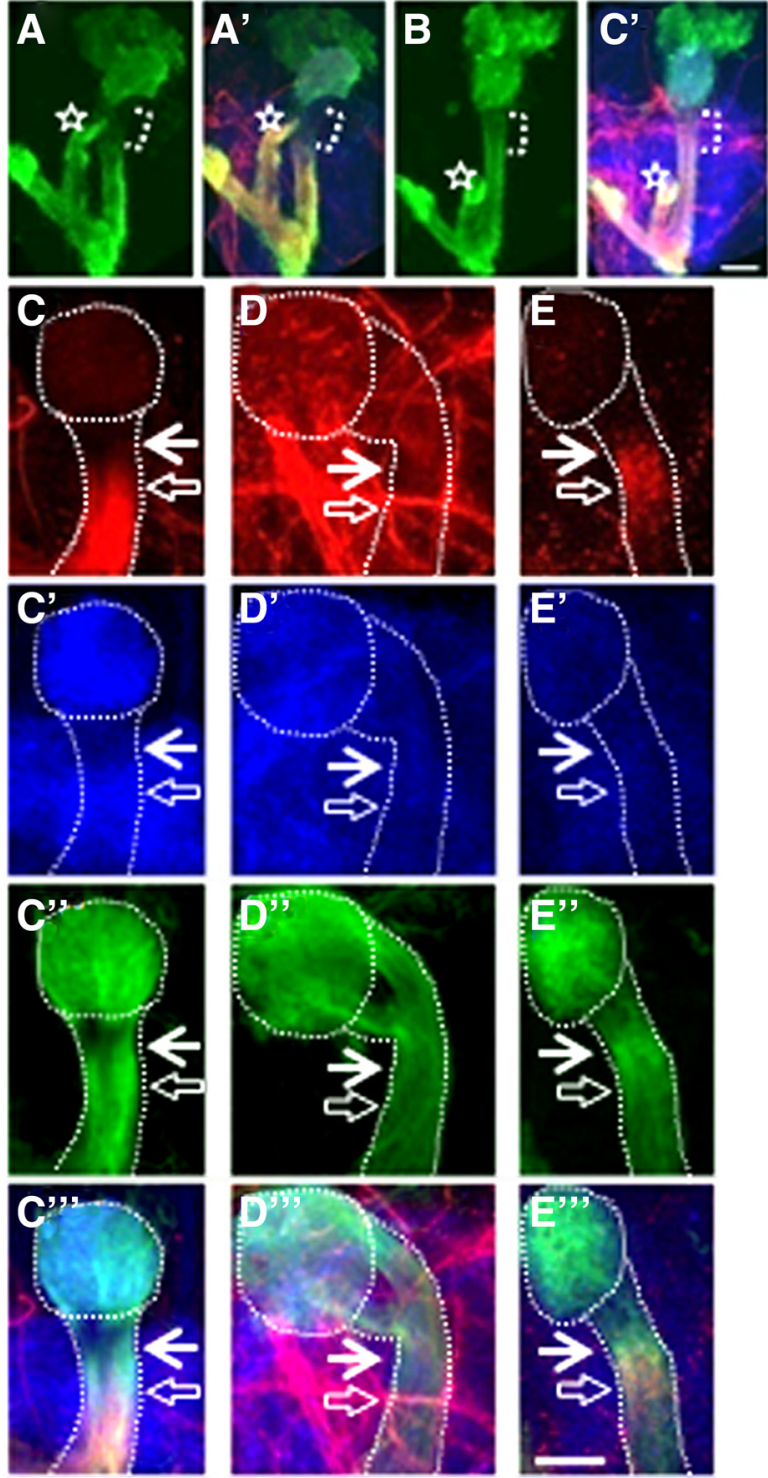

$\mathbf{F}$

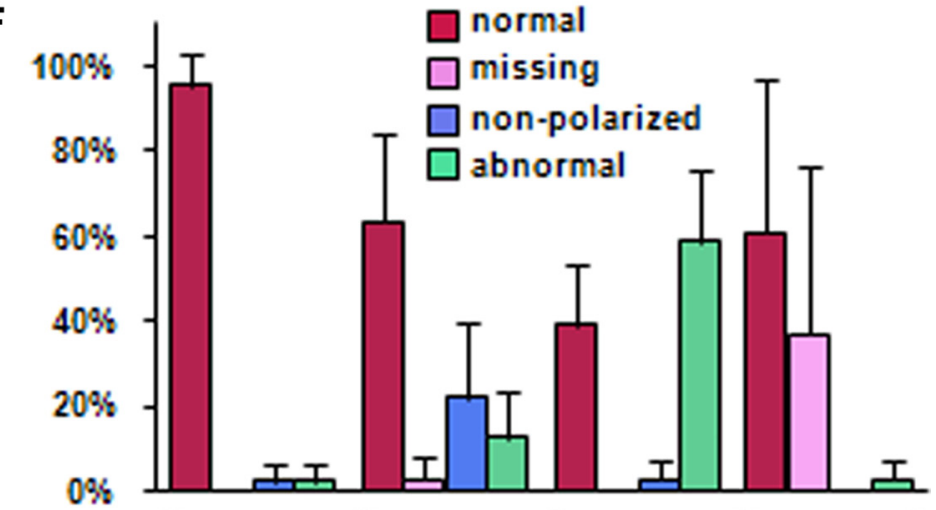

\begin{tabular}{|c|c|c|c|c|}
\hline LatBICytB & - & + & - & + \\
\hline CHAPS & - & - & + & + \\
\hline
\end{tabular}

Figure 3. A dynamic F-actin cytoskeleton controls polarized localization of Fas2. Live brain culturing conditions and staining protocols were as described in Materials and Methods; identical confocal settings were used to show actin cytoskeleton morphology $(\boldsymbol{A}, \boldsymbol{B})$ or analyze Fas 2 patterns $(\boldsymbol{C}-\boldsymbol{F})$. All images are merged $Z$-projections of confocal sections through the whole MB structure $(\boldsymbol{A}, \boldsymbol{B})$ or peduncle $(\boldsymbol{C}-\boldsymbol{E})$ of middle third-stage larvae. $\boldsymbol{A}, \boldsymbol{A}^{\prime}$, Distribution of 201Y::actin-GFP in $\gamma$-neurons after in vitro culturing
NrCAM [neuron-glia cell adhesion molecule (NgCAM)-related cell adhesion molecule], and L1/NgCAM from the L1 CAM family (Winckler et al., 1999; Boiko et al., 2007). This is revealed by altered distribution and sensitivity to detergent extraction of these proteins in the AIS upon treatment with agents preventing actin polymerization (Winckler et al., 1999; Nakada et al., 2003). As a more stringent test of the similarity between the mammalian AIS and the AIS-like compartment of Drosophila $\gamma$-neurons, we applied similar experimental manipulations to cultured larval Drosophila brains and monitored the subcellular localization of the endogenous NCAM (neural cell adhesion molecule)-like protein, Fas2 (Kristiansen and Hortsch, 2008). Similar to mammalian studies, these experiments were done in the presence of cycloheximide to avoid insertion of newly synthesized protein, and rather to ensure that we were tracking preexisting protein. Our live brain culturing condition did not affect general MB morphology but shifted the proximal border of the Fas2-positive axonal signal slightly to-

condition. Note that actin clear zone is not devoid of actin; it is simply at a lower level (compare Fig. 10) $\boldsymbol{B}, \boldsymbol{B}^{\prime}$, Treatment with $0.4 \mu \mathrm{g} / \mathrm{ml}$ LatB was applied for $16 \mathrm{~h}$. Actin-GFP is shown in green; Fas2, in red; and N-Cad, in blue. Bracket shows position of actin-GFP clear zone in controls and equivalent position after LatB treatment. Note the substantial equalization of GFP brightness between AIS and axonal compartments after LatB treatment. The dorsal lobe is marked with a white star. Scale bar, $30 \mu \mathrm{m}$. Effect of pharmacological treatments on Fas2 $\left(\boldsymbol{C}-\boldsymbol{E}\right.$, red) and Ankyrin-GFP $\left(\boldsymbol{C}^{\prime \prime}-\boldsymbol{E}^{\prime \prime}\right.$, green) distribution, $\mathrm{N}$-Cad is shown as a general marker ( $\boldsymbol{C}^{\prime}-\boldsymbol{E}^{\prime}$, blue), and the merge is in $C^{\prime \prime \prime}-E^{\prime \prime \prime}$. The white dotted lines highlight position of calyx and peduncle; the filled white arrow points to proximal and hollow white arrow to distal border of AIS $(\boldsymbol{C}, \boldsymbol{D})$. C, Brain culturing conditions with cycloheximide shifted the proximal Fas2 boundary closer to somatodendritic compartment in $50 \%$ of wild-type brains. This was considered the normal polarized distribution under these conditions. $\boldsymbol{D}$, LatB/ CytB treatment abolished the polarized distribution of Fas2, rendering it diffused and present in all compartments. $\boldsymbol{E}$, CHAPS treatment revealed tethered Fas2 in AIS vicinity. $C^{\prime}-$ $\boldsymbol{E}^{\prime}$, NCad was not affected by LatB/CytB ( $\left.\boldsymbol{C}^{\prime}-\boldsymbol{E}^{\prime \prime}\right)$; Ank1-GFP $\left(C^{\prime}-E^{\prime \prime}\right)$ was randomized by LatB/CytB but not affected by CHAPS. Scale bar, $20 \mu \mathrm{m}$. $\boldsymbol{F}$, Quantification of results from pharmacology. After treatment with indicated drugs, MBs were assigned to one of four phenotypic classes: 1, normal polarized Fas2 distribution (red bar); 2 , missing Fas 2 staining (pink bar); 3, nonpolarized, diffused Fas2 staining (blue bar); and 4 , abnormally polarized Fas 2 accumulated in the vicinity of AIS (green bar). Destabilization of F-actin with LatB/CytB caused diffusion of Fas2, or in combination with CHAPS treatment, prevented Fas 2 accumulation in the interstitial region. Quantification of multiple polarity phenotypes as well as normal morphology is shown as a percentage from total number of MBs ( \pm SD; $n=57-65$ obtained in 3 independent experiments). 


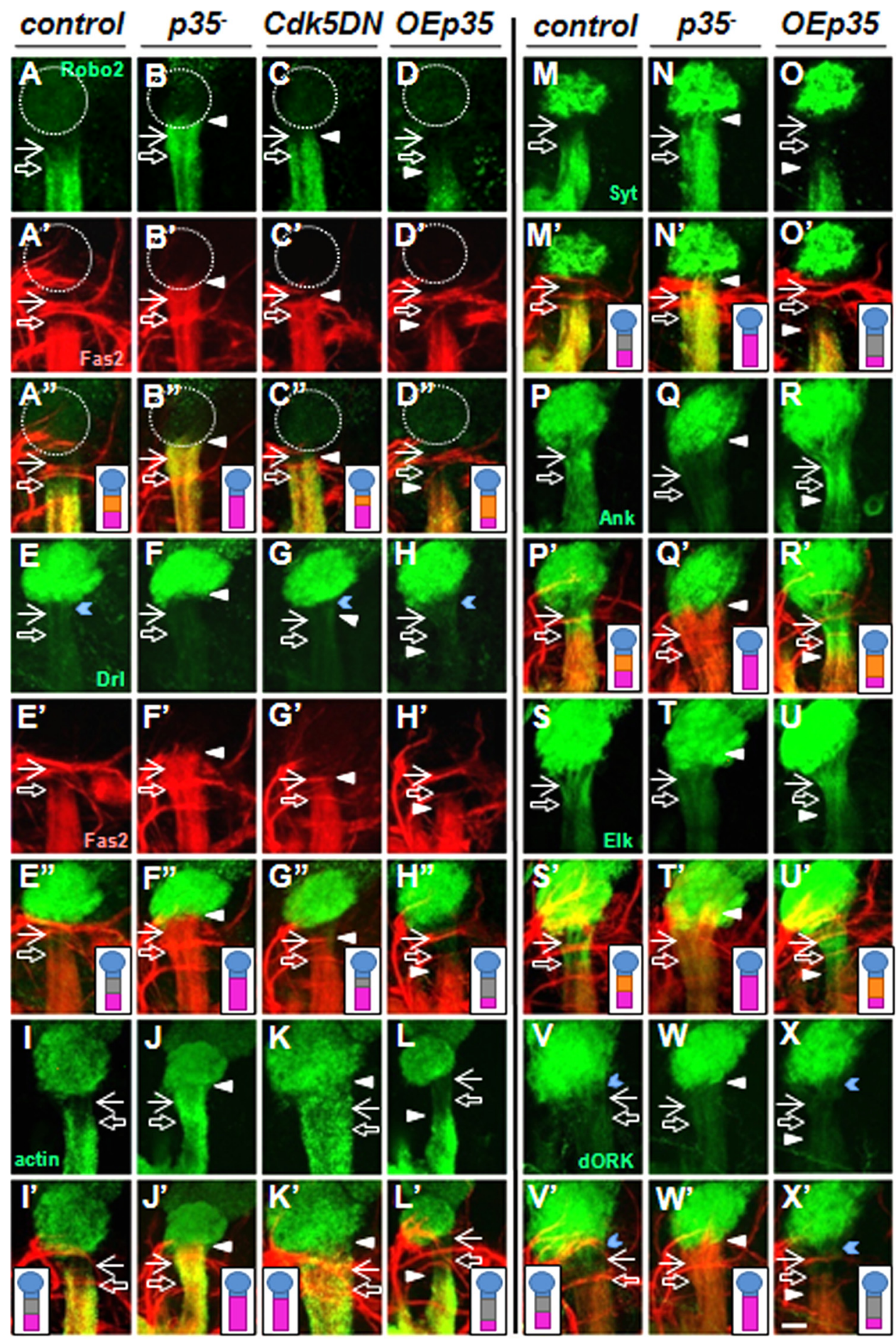

Figure 4. Drosophila Cdk5/p35 kinase regulates the size of the AIS-like compartment. Composite confocal images show the morphology of entire peduncle and calyx of MB at late third larval stage. The solid white arrow points to proximal and the hollow white arrow to distal border of AIS in control MBs and equivalent position in mutants; the white arrowhead points to the shifted position of distal AIS/proximal axon border in mutants. Control brains show polarized distribution of the axonal markers Rob02-GFP and Fas2 $\left(\boldsymbol{A}, \boldsymbol{A}^{\prime}\right)$; and the somatodendritic markers Drl-GFP $(\boldsymbol{E})$ and dORK-C2-GFP $(\boldsymbol{V})$, blue chevron points to their presence beyond calyx; accumulation in the AIS of Ank1-GFP $(\boldsymbol{P})$ and Elk-GFP $(\boldsymbol{S})$; and absence from the AIS of actin-GFP and Syt-HA $(\boldsymbol{I}, \mathbf{M})$. p35 $5^{-}$null transheterozygote mutant and overexpression of UAS-Cdk5DN under 201Y-Gal4 driver (OECdk5DM) have similar phenotypes where position of axonal border was shifted proximally (Rob02 in $\boldsymbol{B}$ and $\boldsymbol{C}$; Fas2 in all backgrounds). Position of distal dendritic compartment border was not affected (Drl in $\boldsymbol{F}$ and $\mathbf{G}$; dORK-C2 in $\boldsymbol{W}$ ). AIS-specific markers failed to accumulate [Ank1 ( $\boldsymbol{Q})$ and Elk $(\boldsymbol{T})$ ] and "clear zones" of AIS-excluded markers failed to develop [actin-GFP ( $\boldsymbol{J}$, $\boldsymbol{K})$ and Syt-HA (N)]. Overexpression of UAS-p35-myc (OEp35) caused a distal shift of axonal markers Rob02 and Fas2 ( $\left.\boldsymbol{D}, \boldsymbol{D}^{\prime}\right)$, while dendritic border was not affected (Drl in $\boldsymbol{H}$ ). The region of accumulation of AIS markers extended down the peduncle [Ank1 $(\boldsymbol{R})$ and Elk $(\boldsymbol{U})$ ], as did clear zones of AlS-excluded markers [actin-GFP $(\boldsymbol{L})$ and syt-HA $(\boldsymbol{O})$ ]. The diagram insets schematically represent $\gamma$-neurons in calyx and peduncle of MB; somatodendritic part is shown in blue; axonal part, in purple; positive AIS labeling, in orange; and absence of a marker in AIS area, in gray. Scale bar: $X^{\prime}, 20 \mu \mathrm{m}$.

ward the cell soma (Fig. 3C). This shifted position is taken as the reference for interpreting the effects of manipulations described below.

First, culturing brains for $16 \mathrm{~h}$ in the presence of a combination of LatB and CytB led to partial randomization of Fas2 localization across the AIS border. Thus, Fas2 protein was now found uniformly distributed across the AIS and the dendrites, as well as the axon (Fig. $3 D, F)$. This demonstrated that active actin polymerization is required to exclude an 
axon-specific protein from the AIS-like and somatodendritic compartments.

Second, extraction of cultured brains with the ionic detergent CHAPS led to Fas 2 accumulation in the vicinity of the AIS in $60 \%$ of MBs (Fig. $3 E, F$ ), revealing the existence of cryptic, detergentresistant Fas 2 tethering sites in the putative AIS. In contrast, such accumulation was not observed if CHAPS extraction was performed in conjunction with inhibition of actin polymerization with LatB/CytB (Fig. 3F). In other words, active polymerization of the actin cytoskeleton was essential to produce CHAPSresistant Fas2 tethering in the AIS. The tethering of Fas2 was specific since another membrane protein, Drosophila N-Cadherin, showed neither accumulation in the AIS in response to CHAPS alone nor distribution changes after inhibiting actin polymerization (Fig. 3 $C^{\prime}, D^{\prime}, E^{\prime}$ ).

Both these observations are analogous to the effects seen in cultured mammalian neurons subjected to similar pharmacological treatments. The finding that the response of protein distribution to complex pharmacological manipulations is conserved in the AIS-like domain of Drosophila $\gamma$-neurons suggests that the fly and vertebrate AIS-like compartments share a deep similarity in the mechanisms of protein tethering that underlie their specialized properties.

\section{Cdk5/p35 kinase regulates the size of the AIS}

As part of a larger effort to characterize Cdk5 functions in neuronal organization, we found that it is a dose-dependent regulator of the length of the AIS-like compartment of $\gamma$-neurons. In p35-null mutant larvae, the proximal boundary of axon markers was shifted substantially closer to the dendrite emergence zone, up to, or even slightly beyond, the position of the normal somatodendritic/AIS boundary, as assayed either with expressed Robo2-GFP and Robo3-GFP markers or endogenous Fas2 (Fig. $\left.4 B, B^{\prime}\right)$. In contrast, the distribution of the somatodendritic markers Drl-GFP, dORK-C2-GFP, and RDL-HA was not affected significantly in $p 35$-null mutants (Fig. $4 F, W$ ), although levels of Drl-GFP and dORK-C2-GFP accumulation between the calyx and compartment border were often reduced. Together, the net effect of these shifts was to shorten or eliminate the AIS-like compartment. Overexpression of a dominant-negative Cdk5 (Connell-Crowley et al., 2000) under the control of various neuronal Gal4 drivers, including the pan-neuronal Elav-GAL4, and $\gamma$-neuron-specific OK107-GAL4, or 201Y-Gal4, produced phenotypes very similar to the $p 35$ mutant, albeit with reduced penetrance and magnitude of shift (Fig. 4C,G,K).

We next examined markers for the AIS-like compartment itself to test directly whether reduction of Cdk5 activity affects its borders. Elk-GFP and ShawDN-GFP, which in wild-type thirdinstar larvae are present both in the somatodendritic compartment and AIS, extend only as far as the border of the somatodendritic compartment in $p 35$ mutants (Fig. $4 S, T$ ). Moreover, the specific accumulation of Ank1-GFP in the AIS is completely absent as are the AIS-specific "clear zones" of Syt-HA, Syt-GFP, and brp-GFP, and the zone of specialized F-actin (Fig. $4 Q, N, J$ ). The proximal shift in the border of Fas2 accumulation was also observed in a null mutant for Cdk5 (Kissler et al., 2009) (data not shown). These data suggest that, in the absence of Cdk5 activity, the distal border of the AIS-like compartment shifts dramatically toward the cell soma with reduction or elimination of the AIS, in parallel with a corresponding proximal shift in the border of the axonal compartment.

Increasing Cdk 5 activity by overexpression of wild-type $p 35$ (Connell-Crowley et al., 2000) had an effect opposite to that of the loss of function. The border of the axonal compartment defined by the markers Fas2, Robo2-GFP, and Robo3-GFP was shifted further away from the somatodendritic compartment (Fig. $4 D, D^{\prime}$ ). For example, when p35 expression was driven by $201 Y$-GAL4, the proximal boundary of axon markers was located $65 \mu \mathrm{m}$ away from the point of dendrite emergence, in contrast to $41 \mu \mathrm{m}$ in controls (Fig. 2). Similar to the loss-of-function experiments, the distribution of the somatodendritic-specific markers Drl-GFP, dORK-C2-GFP, and RDL-HA was not affected by $p 35$ overexpression (Fig. $4 H, X$ ). Consistent with these changes in the boundaries of somatodendritic and axonal markers, the length of the putative AIS itself was substantially increased by Cdk 5 hyperactivity. Thus, the distal border of Elk-GFP moved distally in register with the shift in axonal markers (Fig. $4 U$ ), and markers that define both ends of the AIS [Ank1-GFP accumulation, the Syt-HA clear zone, and the zone of altered actin-GFP (Fig. $4 L, O, R)]$ showed an equivalent increase in length due to a shift of the distal boundary.

Three lines of experiments suggested that alterations of the AIS-like compartment upon loss of Cdk5 activity were not secondary to disruption of the general morphogenesis and organization of MB neurons. First, we generated single, marked p35 mutant cells in a nonmutant genetic background [by MARCM (Lee and Luo, 1999)] or labeled single cells in uniformly mutant p35 $5^{-}$brains [with FLP-out (Wong et al., 2002)]. In both cases, p35 mutant MB neurons had normal morphology in terms of initial establishment of dendrites and axons, dendrite branching, and axon targeting (Fig. 5). Second, the segregation of markers between the axonal and somatodendritic compartment was not compromised; we did not observe gross relocalization of markers in the mutant suggestive of loss of the fundamental polarization of the neuron. Third, one could postulate that movement of the Fas 2 border might occur due to a change in the fasciculation of the neurites coming from the four MB lineages. However, examination of large MARCM $p 35^{-}$clones and FLP-out clones in the p35 mutant showed normal fasciculation of these projections even when the Fas2 border was significantly shifted (data not shown).

\section{The AIS-like compartment and its regulation by $\mathrm{Cdk} 5$ are maintained in the adult}

Extending our analysis of $\gamma$-neuron subcellular organization to the adult brain revealed that both the presence of an AIS-like compartment and its regulation by Cdk 5 activity persist into the adult. MB neurons, including $\gamma$-neurons, are extensively remodeled at metamorphosis to generate new dendritic arbors and altered axonal targeting. Three features were used to identify $\gamma$-neurons in the adult brain: expression of the 201Y-Gal4 driver (also present in a subset of $\alpha$-, $\beta$-neurons in adults) (Fig. 6 $\mathrm{A}^{\prime \prime}-$ $C^{\prime \prime}, D-L$, white bracket) (Kurusu et al., 2002), position of projections in the inner core of the peduncle (Kurusu et al., 2002), and low level expression of Fas2 $\left(A^{\prime}-C^{\prime}\right)$ (Ng et al., 2002). Applying these criteria, we found that the Fas 2 pattern changes in $\mathrm{MB}$ neurons after the pupal stage. Adult $\gamma$-neurons express Fas2 at low level but keep a subcellular distribution of Fas2 similar to that in larvae. In contrast, adult $\alpha$ - and $\beta$-neurons turn on Fas 2 at high level and here Fas2 has a novel localization pattern, different from that of $\gamma$-neurons.

Examining in the adult the same markers we had characterized in the larva revealed an interstitial domain in wild-type $\gamma$-neurons. This domain intervenes between the somatodendritic compartment (Drl-GFP, dORK-C2-GFP) and axonal compartment (Fas2, Robo2-GFP and Robo3-GFP) (Fig. 6A, $A^{\prime}$ ). This 
A

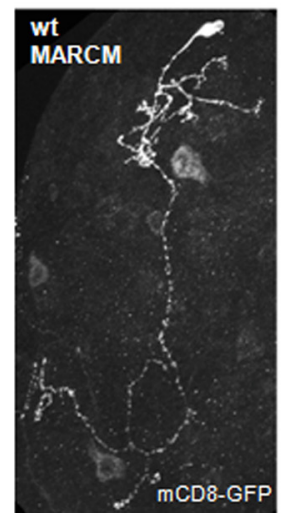

B

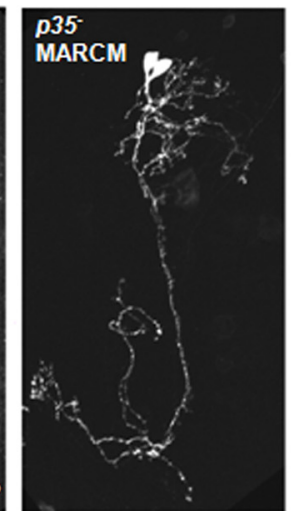

C

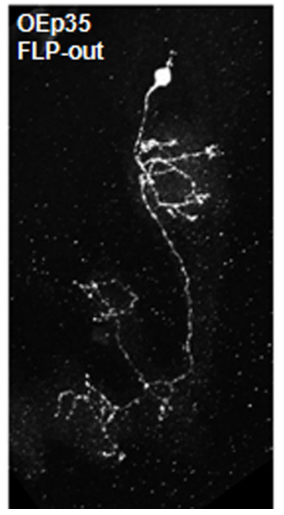

D

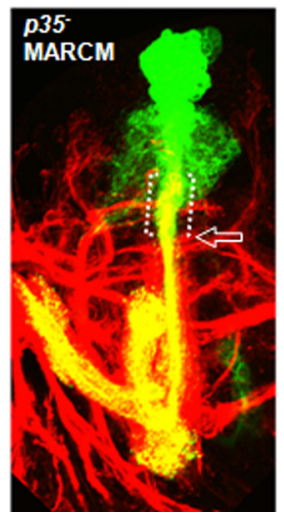

D'

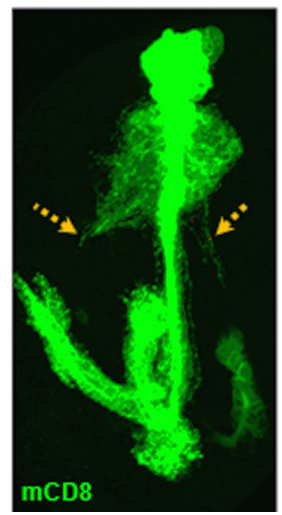

D"

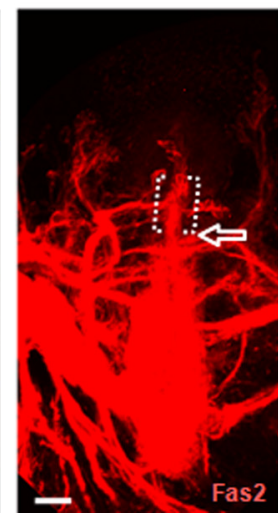

Figure 5. Neuronal morphology is not affected by altered Cdk/p35 activity in $\gamma$-neurons. $\boldsymbol{A}-\boldsymbol{C}$, Control MARCM one cell clone (A), p35 mutant MARCM two cell clone (B), and OEp35 Flip-0ut one cell clone $(\boldsymbol{C})$ all have wild-type morphology: axons and dendrites differentiate properly and project to correct target areas (control and $p 35$ mutant Flip-out clones also exhibit wild-type morphology) (data not shown). For orientation details, see Figure 1/legend. D, Big p35 mutant MARCM clone included the whole lineage of one neuroblast and show a subtle dendritic mistargeting phenotype $\left(\boldsymbol{D}^{\prime}\right.$, orange dotted arrow). Dendritic mistargeting was observed at low frequency $(7 \%)$ in single cell clones $(n=70)$ and will be described in detail elsewhere. Note that Fas 2 border is shifted proximally in the bundle of axons coming from big $p 35$ mutant clone (brackets show shifted position above control level marked by white hollow arrow in $\boldsymbol{D}$ and $\boldsymbol{D}^{\prime \prime}$ ). $\mathrm{mCD}$-GFP is shown in green, and Fas2, in red. Scale bar, $20 \mu \mathrm{m}$.

novel compartment accumulated Ank1-GFP, excluded synaptic vesicle components (Syt-HA and Syt-GFP), had an altered actin composition (actin-GFP), and had the same unique combination of K-channel markers as the larval AIS (Fig. 6D, G,J). The putative adult AIS was slightly longer than that of the larva, $26 \pm 3$ $\mu \mathrm{m}$, remarkably in close agreement with the size of a typical vertebrate AIS [25-45 $\mu \mathrm{m}$ (Van Wart et al., 2007)] despite the far greater size of vertebrate neurons.

We also found that the function of Cdk5/p35 kinase in AIS organization was preserved in adult neurons. As in the larva, reduced Cdk5 activity caused the AIS/axonal compartment boundary to shift proximally, with corresponding shortening or absence of the AIS (Fig. $6 B, E, H, K$ ), and with axonal markers sometimes spreading into the region between the proximal AIS boundary and the zone of dendrite emergence. Conversely, increased Cdk5 activity led to a distal shift of this boundary, associated with increased AIS length (Fig. 6C,F,I,L).

Finally, we analyzed the distribution of Cdk 5 and $\mathrm{p} 35$ proteins at late larval and adult stages by constitutively expressing tagged forms of the proteins in $\gamma$-neurons. In larval $\gamma$-neurons, p35-myc was enriched in the somatodendritic compartment, AIS, and the peduncle portion of the axon, and was present at low level in the distal axonal lobes (Fig. $7 A-A^{\prime \prime}$ ). In adult neurons, this pattern was modified, now being low throughout both the peduncle and lobar regions of the axon, but remaining enriched in the somatodendritic compartment and AIS (Fig. 7C$\left.C^{\prime \prime}\right)$. Both Cdk5-FLAG wild-type and dominant-negative forms had identical patterns and were equally distributed through all neural compartments at late larval and adult stages, consistent with previously published data (Fig. 7B$\left.B^{\prime \prime}, D-D^{\prime \prime}\right)$ (Connell-Crowley et al., 2000; Hahn et al., 2005). Based on distribution of tagged derivatives, these data suggest that both Cdk5 subunits are likely to be present in the AIS compartment both during development and adulthood.

\section{Discussion}

The axon initial segment is the specialized domain of a vertebrate neuron where action potentials initiate. It lies between the axonal and somatodendritic compartments of the cell, acting as a gatekeeper between the two, and is characterized by diagnostic mo- lecular features. In this work, we show that Drosophila $\gamma$-neurons have a compartment in the proximal axon that shares many of the key hallmarks of the vertebrate AIS. The putative Drosophila AIS lies between the axonal and somatodendritic compartments and fails to label with markers for either one. It has a unique actin organization revealed structurally by labeling with actin-GFP and functionally by challenging with a variety of pharmacological treatments, and it accumulates a specific ankyrin isoform, Ankyrin 1. Finally, it localizes markers for a unique combination of potassium channels, consistent with having a unique electrophysiological character. We demonstrate that the protein kinase C $\mathrm{dk} 5$ is a dose-dependent regulator of the structure of this compartment: flies with increased Cdk5 activity have a longer AIS; those with reduced activity have a shorter AIS or none at all, both in developing larvae and in the adult.

Drosophila is a powerful model for understanding many fundamental neuronal properties but one central aspect, subcellular organization, has resisted analysis in the fly due to size limitations on electrophysiology, and the lack of reagents for localizing sodium channels. We therefore performed a systematic analysis of multiple molecular markers to examine the structural features of Drosophila central neurons and discovered a specialized axonal compartment that is analogous or homologous to the vertebrate AIS. Recent results from other laboratories are consistent with this interpretation. Analysis of cultured Drosophila motoneurons has revealed that lateral diffusion of membrane proteins is greatly restricted in a $\sim 20 \mu \mathrm{m}$ band that lies near the junction between the somatodendritic and axonal compartments (Katsuki et al., 2009). Moreover, Rolls et al. (2007) have speculated that Drosophila axons may have an AIS-like domain based on localization of expressed chick NgCAM and fly APC2 in a portion of the proximal axon, although they did not test this idea further. Finally, the action potential initiation zone of Drosophila antennal lobe projection neurons has recently been mapped to the proximal axon, beyond the site of dendrite emergence, analogous to where we map the AIS in MB neurons (Gouwens and Wilson, 2009). In preliminary experiments, we find an interstitial domain in antennal lobe projection neurons, as well as some subclasses of $\mathrm{MB} \alpha$ - and $\beta$-neurons, and confirm their ability to accumulate at 

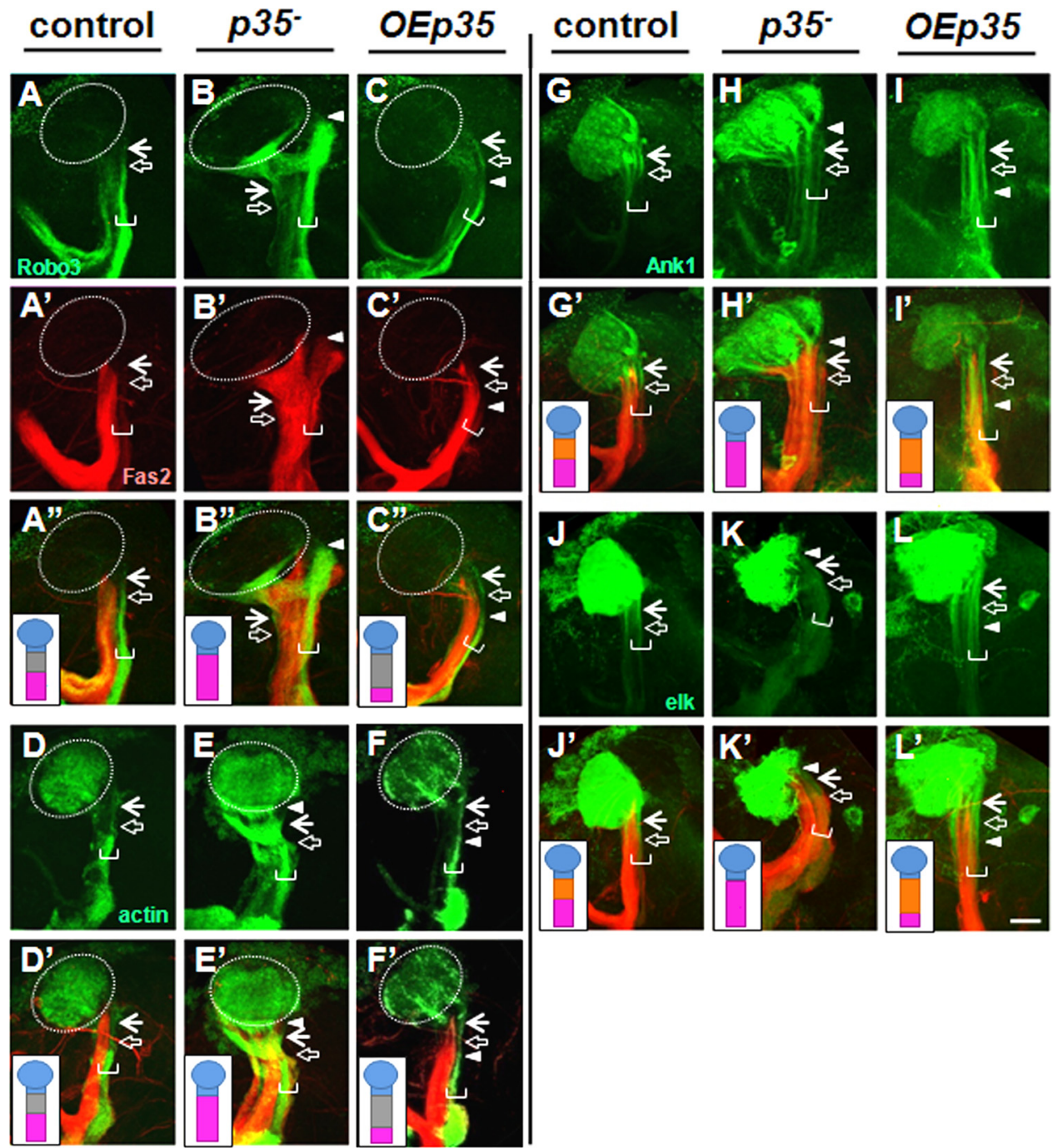

Figure 6. C $\mathrm{dk} 5 / \mathrm{p} 35$ kinase controls the maintenance of AIS-like compartment in adult neurons. Examination of markers at the adult stage revealed an interstitial domain in wild-type $\gamma$-neurons. For symbol definitions, see the legend of Figure 4. The horizontal white bracket indicates $\gamma$-neurons. AlS compartment intervenes between the axonal Fas2/Robo3-positive $\left(\boldsymbol{A}, \boldsymbol{A}^{\prime}, \boldsymbol{A}^{\prime \prime}\right)$ and somatodendritic Drl-positive compartments (data not shown), is positively marked by accumulation of Ank1-GFP (G), Elk potassium channel (D), and clear zone of actin-GFP distribution (D). The putative AIS was $\sim 26 \pm 3$ - $\mu \mathrm{m}$-long $\left(n=6\right.$ for Ank1-GFP marker) in young adult males. p35-null mutant neurons shifted axonal boundary proximally $\left(\boldsymbol{B}, \boldsymbol{B}^{\prime}, \boldsymbol{B}^{\prime \prime}\right)$ and abolished AIS-specific clearance of actin-GFP $(\boldsymbol{E})$, and accumulation of Ank1-GFP $(\boldsymbol{H})$, and Elk-GFP $(\boldsymbol{K})$. OEp35 shifted axonal boundary $\left(\boldsymbol{C}, \boldsymbol{C}^{\prime}, \boldsymbol{C}^{\prime}\right)$ further from soma and extended AIS length $(\boldsymbol{F}, \boldsymbol{I}, \boldsymbol{L})$, creating $45 \pm 9 \mu \mathrm{m}$ $(n=7$ for Ank1-GFP) compartment. Fas2 staining is shown in red, and the corresponding UAS construct expressed under 201Y-Gal4, in green. Scale bar, $30 \mu \mathrm{m}$.

least some of the markers characterized here for $\gamma$-neurons (S. Trunova and E. Giniger, unpublished observations).

It is perhaps not surprising that Drosophila central brain neurons form a demarcation between the axonal and somatodendritic membranes, much as do motoneurons (Katsuki et al., 2009). However, given the morphological differences between insect and vertebrate neurons (Sánchez-Soriano et al., 2005; Hill et al., 2008), and in particular given that flies lack a specialized AnkyrinG and AnkG-binding motifs in voltage-gated $\mathrm{Na}$ and $\mathrm{K}$ channels (Hill et al., 2008), it was completely unexpected that Drosophila neurons would possess a compartment with such striking similarity to the vertebrate AIS. It is formally possible that this AIS-like compartment reflects a remarkable example of parallel evolution between invertebrates and vertebrates, but the simplest interpretation is that the vertebrate and Drosophila AIS are homologous structures, derived from a specialized compartment already present in the common predecessor to vertebrates and invertebrates (Rasband, 2010) and able to localize voltage- gated channels using AnkyrinG-independent mechanisms. Our results thus offer the opportunity to investigate molecular mechanisms of AIS development and regulation using the simpler Drosophila model.

Several lines of evidence suggest that regulation of AIS size is likely to be a direct function of Cdk5 rather than a secondary consequence of nonspecific perturbations of neuronal structure. First, we observed a proximal shift in the border of axon-specific markers in $\gamma$-neurons as early as the second larval instar, after connections are made but while the brain is still growing in size (data not shown). Second, Cdk5 did not produce any gross change in the fundamental axo/dendritic polarization of $\gamma$-neurons; compartment-specific molecular markers retained their overall distribution, although in some contexts axonal markers spread slightly into the distal portion of the somatodendritic domain. Third, the overall morphology of the MB and the stereotyped pattern of axonal and dendritic projections of individual neurons were not disturbed by altered Cdk5 activity. Fourth, the 


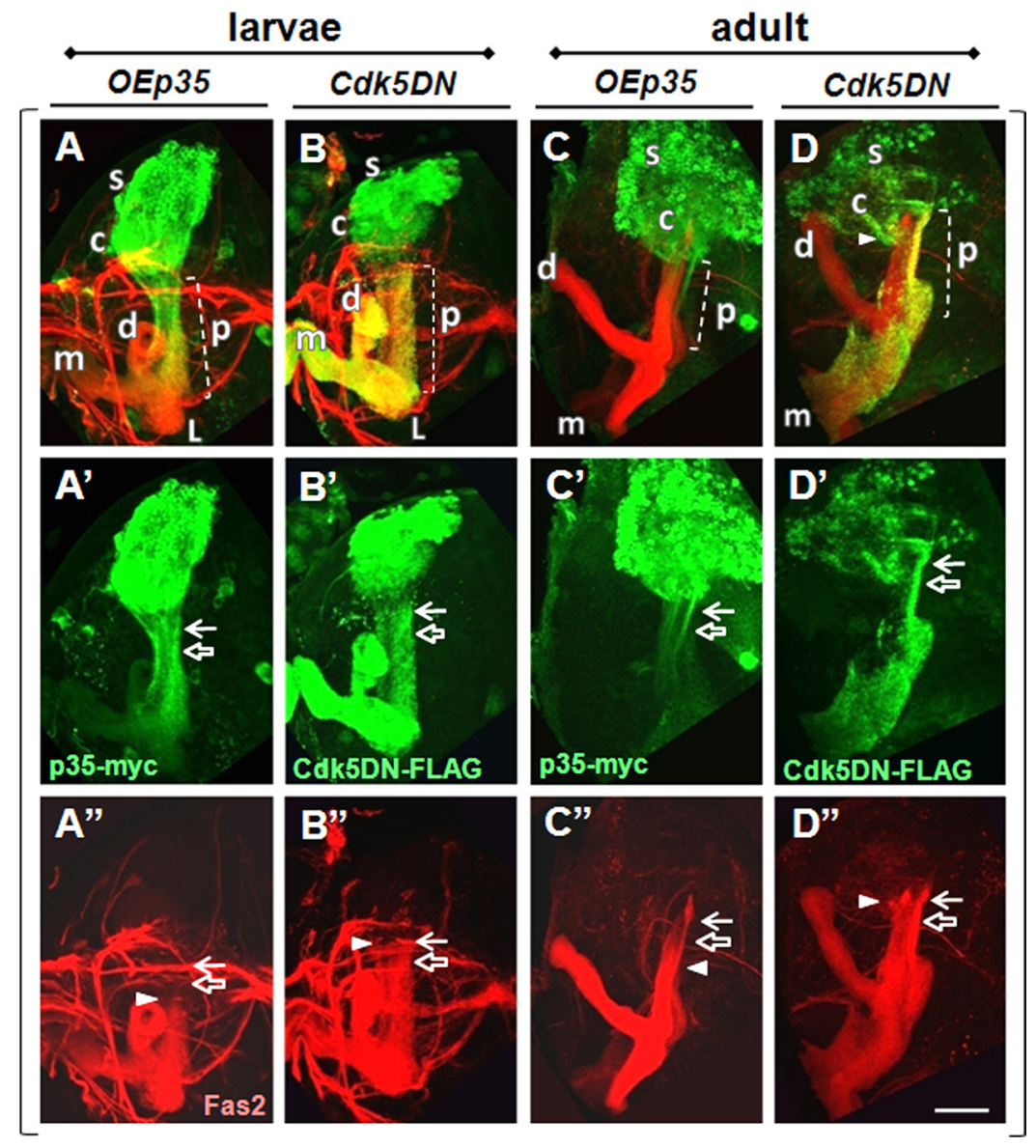

Figure 7. Intraneuronal distribution of $\mathrm{p} 35$ and Cdk5 tagged proteins. At larval stage, $\mathrm{p} 35$-myc is enriched in soma, calyx, and peduncle $\left(A, A^{\prime}\right)$, while Cdk5-FLAG wild-type and Cdk5-FLAG dominant-negative forms have identical distribution patterns and are present through all parts of neuron $\left(\boldsymbol{B}, \boldsymbol{B}^{\prime}\right)$. At adult stage, p35-myc is enriched in soma, calyx, and AIS and present in axonal part at low level $\left(\boldsymbol{C}, \boldsymbol{C}^{\prime}\right)$. Cdk5 is enriched in soma and axonal part and present at lower level in calyx. $\mathbf{D}^{\prime \prime}$, In adult brain, OECdk5DN has maintained larval phenotype and shifted up the Fas 2 border $\left(\boldsymbol{B}^{\prime \prime}, \boldsymbol{D}^{\prime \prime}\right.$, white arrowhead). The brackets combine images from the same brain. For schematic details, see the legends of Figures 1 and 4 . Scale bar, $30 \mu \mathrm{m}$.

effect of Cdk5 gain and loss of function on AIS length was autonomous to $\gamma$-neurons in various mosaic paradigms and did not require altered Cdk5 function in other neuronal types, or in glia.

It is unlikely that effects of Cdk5 on synaptic function and overall neuronal excitability are responsible for the observed Cdk5-dependent changes in AIS length. Among the markers we expressed to analyze potassium channel distribution were the potassium leak channel dORK (Nitabach et al., 2002), the Shaker derivative electrical knock-out (EKO) (White et al., 2001), and a dominant-negative form of Shaw (Hodge et al., 2005). Expression of dORK and EKO has been used to silence neurons electrically, while expression of dn-Shaw increases neuronal excitability. However, expression of these proteins in wild-type $\gamma$-neurons had at most subtle effects on the position of the AIS boundary, and it did not substantially influence the ability of Cdk5 activity to shift that boundary. This argues that the effect of Cdk5 on AIS length was separate from any baseline change it might cause in neuronal excitability. Moreover, the use of both active and inactive marked transgenes and of multiple, different epitope tags makes it likely that the tagged marker proteins are faithfully reporting the localization of the endogenous proteins.

Both the size and position of the AIS can be modulated, but how are these controlled, and how are they linked to the positioning of adjacent cellular compartments? Our data, together with published results, imply that the position of the two AIS borders can be regulated separately. First, altering Cdk5 activity changed the position of the junction between the AIS and axon proper, but not the AIS/somatodendritic border, just as input deprivation moved only the distal AIS border (Kuba et al., 2010). In contrast, chronic depolarization of cultured hippocampal neurons shifts the position of the AIS without altering its size, implying context-dependent regulation of the positions of both boundaries (Grubb and Burrone, 2010). Second, while we cannot formally exclude the hypothesis that Cdk5 acts separately on each of the proteins we assayed, the simplest hypothesis is that Cdk5 acts near the top of a molecular cascade that coordinately controls the localization of the entire suite of molecules that populate the AIS. All the axonal markers we have analyzed shift in parallel in response to modulation of $\mathrm{Cdk} 5$, and the same is true for all the AIS markers. However, Cdk5 clearly acts downstream of the basic axo/dendritic polarization of the neuron since this is essentially unaffected by gain or loss of Cdk5 activity. In contrast, gross pharmacological disruption of actin structure caused a very different phenotype, eliminating polarized distribution of Fas2. This implies that the effects of Cdk5 on the AIS are not caused simply by overall disorganization of the actin cytoskeleton.

Finally, our results raise the question of how the positions of the borders of the AIS are connected to positioning of the borders of adjacent compartments. One could postulate that each neuronal compartment-somatodendritic, AIS, and axon-is an integrated unit whose boundaries can be modulated independently of the others. We find, however, that Cdk5associated alterations of the AIS are invariably associated with a corresponding shift in the positions of axonal markers, while others have shown that disassembly of the AIS by depletion of AnkG is associated with a distal shift of somatodendritic markers (Hedstrom et al., 2008; Sobotzik et al., 2009). This suggests a tight linkage between the mechanisms controlling the positioning of components in adjacent compartments in vivo.

The AIS-like compartment was found to persist into adulthood in Drosophila $\gamma$-neurons, as was the ability of Cdk5 to modulate it. The length and position of the vertebrate AIS clearly affect neuronal excitability and recent results show that control over these AIS properties is part of the mechanism of plasticity and neuronal homeostasis both in vitro and in vivo (Grubb and Burrone, 2010; Kuba et al., 2010). Given the role of mammalian Cdk5 in synaptic homeostasis (Seeburg et al., 2008), it seems natural to speculate that Cdk5 may also be involved in the mechanism of homeostatic regulation of AIS structure. Moreover, it is striking that the response to various forms of neuronal injury involves calpain-dependent proteolysis of AIS components (Schafer et al., 2009; von Reyn et al., 2009). One key target of calpain is the p35 regulatory subunit of Cdk5. Calpain cleavage 
converts p35 to a deregulated and mislocalized form, p25, leading to excessive and mistargeted Cdk 5 activity. Since Cdk5 is strongly implicated in the initiation and progression of many neurodegenerative diseases, including Alzheimer's, ALS, and Parkinson's, this raises the unexpected possibility that some of the neuronal dysfunction that occurs in response to injury, and perhaps also in Cdk5-associated disease, arises from alterations in spike initiation and neuronal compartmentalization as a consequence of changes in AIS structure or integrity.

\section{References}

Boiko T, Vakulenko M, Ewers H, Yap CC, Norden C, Winckler B (2007) Ankyrin-dependent and -independent mechanisms orchestrate axonal compartmentalization of L1 family members neurofascin and L1/ neuron-glia cell adhesion molecule. J Neurosci 27:590-603.

Bouley M, Tian MZ, Paisley K, Shen YC, Malhotra JD, Hortsch M (2000) The L1-type cell adhesion molecule neuroglian influences the stability of neural ankyrin in the Drosophila embryo but not its axonal localization. J Neurosci 20:4515-4523.

Connell-Crowley L, Le Gall M, Vo DJ, Giniger E (2000) The cyclindependent kinase Cdk5 controls multiple aspects of axon patterning in vivo. Curr Biol 10:599-602.

Connell-Crowley L, Vo D, Luke L, Giniger E (2007) Drosophila lacking the Cdk5 activator, p35, display defective axon guidance, age-dependent behavioral deficits and reduced lifespan. Mech Dev 124:341-349.

Gouwens NW, Wilson RI (2009) Signal propagation in Drosophila central neurons. J Neurosci 29:6239-6249.

Grubb MS, Burrone J (2010) Activity-dependent relocation of the axon initial segment fine-tunes neuronal excitability. Nature 465:1070-1074.

Hahn CM, Kleinholz H, Koester MP, Grieser S, Thelen K, Pollerberg GE (2005) Role of cyclin-dependent kinase 5 and its activator P35 in local axon and growth cone stabilization. Neuroscience 134:449-465.

Hedstrom KL, Rasband MN (2006) Intrinsic and extrinsic determinants of ion channel localization in neurons. J Neurochem 98:1345-1352.

Hedstrom KL, Ogawa Y, Rasband MN (2008) AnkyrinG is required for maintenance of the axon initial segment and neuronal polarity. J Cell Biol 183:635-640.

Hill AS, Nishino A, Nakajo K, Zhang G, Fineman JR, Selzer ME, Okamura Y, Cooper EC (2008) Ion channel clustering at the axon initial segment and node of Ranvier evolved sequentially in early chordates. PLoS Genet 4:e1000317.

Hodge JJ, Choi JC, O’Kane CJ, Griffith LC (2005) Shaw potassium channel genes in Drosophila. J Neurobiol 63:235-254.

Ip NY, Tsai LH (2008) Cyclin-dependent kinase 5 (Cdk5). New York: Springer Science + Business Media.

Katsuki T, Ailani D, Hiramoto M, Hiromi Y (2009) Intra-axonal patterning: intrinsic compartmentalization of the axonal membrane in Drosophila neurons. Neuron 64:188-199.

Kissler AE, Pettersson N, Frölich A, Sigrist SJ, Suter B (2009) Drosophila cdk 5 is needed for locomotive behavior and NMJ elaboration, but seems dispensable for synaptic transmission. Dev Neurobiol 69:365-377.

Ko J, Humbert S, Bronson RT, Takahashi S, Kulkarni AB, Li E, Tsai LH (2001) p35 and p39 are essential for cyclin-dependent kinase 5 function during neurodevelopment. J Neurosci 21:6758-6771.

Kole MH, Ilschner SU, Kampa BM, Williams SR, Ruben PC, Stuart GJ (2008) Action potential generation requires a high sodium channel density in the axon initial segment. Nat Neurosci 11:178-186.

Kristiansen LV, Hortsch M (2008) Fasciclin II: the NCAM ortholog in Drosophila melanogaster. Adv Exp Med Biol 663:387-401.

Kuba H, Oichi Y, Ohmori H (2010) Presynaptic activity regulates $\mathrm{Na}^{+}$ channel distribution at the axon initial segment. Nature 465:1075-1078.

Kurusu M, Awasaki T, Masuda-Nakagawa LM, Kawauchi H, Ito K, Furukubo-Tokunaga K (2002) Embryonic and larval development of the Drosophila mushroom bodies: concentric layer subdivisions and the role of fasciclin II. Development 129:409-419.
Lasiecka ZM, Yap CC, Vakulenko M, Winckler B (2009) Compartmentalizing the neuronal plasma membrane from axon initial segments to synapses. Int Rev Cell Mol Biol 272:303-389.

Lee T, Luo L (1999) Mosaic analysis with a repressible cell marker for studies of gene function in neuronal morphogenesis. Neuron 22:451-461.

Lee T, Lee A, Luo L (1999) Development of the Drosophila mushroom bodies: sequential generation of three distinct types of neurons from a neuroblast. Development 126:4065-4076.

Mosca TJ, Carrillo RA, White BH, Keshishian H (2005) Dissection of synaptic excitability phenotypes by using a dominant-negative Shaker $\mathrm{K}^{+}$ channel subunit. Proc Natl Acad Sci U S A 102:3477-3482.

Nakada C, Ritchie K, Oba Y, Nakamura M, Hotta Y, Iino R, Kasai RS, Yamaguchi K, Fujiwara T, Kusumi A (2003) Accumulation of anchored proteins forms membrane diffusion barriers during neuronal polarization. Nat Cell Biol 5:626-632.

Ng J, Nardine T, Harms M, Tzu J, Goldstein A, Sun Y, Dietzl G, Dickson BJ, Luo L (2002) Rac GTPases control axon growth, guidance and branching. Nature 416:442-447.

Nitabach MN, Blau J, Holmes TC (2002) Electrical silencing of Drosophila pacemaker neurons stops the free-running circadian clock. Cell 109: 485-495.

Ogawa Y, Rasband MN (2008) The functional organization and assembly of the axon initial segment. Curr Opin Neurobiol 18:307-313.

Pielage J, Cheng L, Fetter RD, Carlton PM, Sedat JW, Davis GW (2008) A presynaptic giant ankyrin stabilizes the NMJ through regulation of presynaptic microtubules and transsynaptic cell adhesion. Neuron 58: 195-209.

Rasband MN (2010) The axon initial segment and the maintenance of neuronal polarity. Nat Rev Neurosci 11:552-562.

Rolls MM, Satoh D, Clyne PJ, Henner AL, Uemura T, Doe CQ (2007) Polarity and intracellular compartmentalization of Drosophila neurons. Neural Dev 2:7.

Ryglewski S, Duch C (2009) Shaker and Shal mediate transient calciumindependent potassium current in a Drosophila flight motoneuron. J Neurophysiol 102:3673-3688.

Sánchez-Soriano N, Bottenberg W, Fiala A, Haessler U, Kerassoviti A, Knust E, Löhr R, Prokop A (2005) Are dendrites in Drosophila homologous to vertebrate dendrites? Dev Biol 288:126-138.

Schafer DP, Jha S, Liu F, Akella T, McCullough LD, Rasband MN (2009) Disruption of the axon initial segment cytoskeleton is a new mechanism for neuronal injury. J Neurosci 29:13242-13254.

Seeburg DP, Feliu-Mojer M, Gaiottino J, Pak DT, Sheng M (2008) Critical role of CDK5 and Polo-like kinase 2 in homeostatic synaptic plasticity during elevated activity. Neuron 58:571-583.

Silies M, Klämbt C (2010) APC/C(Fzr/Cdh1)-dependent regulation of cell adhesion controls glial migration in the Drosophila PNS. Nat Neurosci 13:1357-1364.

Sobotzik JM, Sie JM, Politi C, Del Turco D, Bennett V, Deller T, Schultz C (2009) Ankyring is required to maintain axo-dendritic polarity in vivo. Proc Natl Acad Sci U S A 106:17564-17569.

Van Wart A, Trimmer JS, Matthews G (2007) Polarized distribution of ion channels within microdomains of the axon initial segment. J Comp Neurol 500:339-352.

von Reyn CR, Spaethling JM, Mesfin MN, Ma M, Neumar RW, Smith DH, Siman R, Meaney DF (2009) Calpain mediates proteolysis of the voltage-gated sodium channel alpha-subunit. J Neurosci 29:1035010356.

White BH, Osterwalder TP, Yoon KS, Joiner WJ, Whim MD, Kaczmarek LK, Keshishian H (2001) Targeted attenuation of electrical activity in Drosophila using a genetically modified $\mathrm{K}^{+}$channel. Neuron 31:699-711.

Winckler B, Forscher P, Mellman I (1999) A diffusion barrier maintains distribution of membrane proteins in polarized neurons. Nature 397: 698-701.

Wong AM, Wang JW, Axel R (2002) Spatial representation of the glomerular map in the Drosophila protocerebrum. Cell 109:229-241. 